\title{
Electrical pumping of potassium ions against an external concentration gradient in a biological ion channel
}

\author{
María Queralt-Martín, ${ }^{1}$ Elena García-Giménez, ${ }^{1}$ Vicente M. Aguilella, ${ }^{1}$ Patricio Ramirez, ${ }^{2}$ \\ Salvador Mafe, ${ }^{3}$ and Antonio Alcaraz ${ }^{1, a)}$ \\ ${ }^{1}$ Laboratorio de Biofísica Molecular, Departament de Física, Universitat Jaume I, E-12080 Castellón, Spain \\ ${ }^{2}$ Departament de Física Aplicada, Universitat Politécnica de València, E-46022 València, Spain \\ ${ }^{3}$ Departament de Física de la Terra i Termodinàmica, Universitat de València, E-46100 Burjassot, Spain
}

(Received 7 June 2013; accepted 9 July 2013; published online 26 July 2013)

\begin{abstract}
We show experimentally and theoretically that significant currents can be obtained with a biological ion channel, the OmpF porin of Escherichia coli, using zero-average potentials as driving forces. The channel rectifying properties can be used to pump potassium ions against an external concentration gradient under asymmetric $\mathrm{pH}$ conditions. The results are discussed in terms of the ionic selectivity and rectification ratio of the channel. The physical concepts involved may be applied to separation processes with synthetic nanopores and to bioelectrical phenomena. (C) 2013 AIP Publishing LLC. [http://dx.doi.org/10.1063/1.4816748]
\end{abstract}

Ratchet systems have the ability of rectifying unbiased fluctuations into directed transport. ${ }^{1-3}$ Both synthetic and biological nanochannels may show non-zero electrical currents driven by zero-average time dependent forces. ${ }^{4-7}$ The electrical rectification is on the basis of the observed phenomena $^{4,5,8,9}$ and the potential applications concern separation processes and energy conversion. ${ }^{10-12}$ Electrical rectification and ratchet phenomena can also be of relevance for signal averaging of weak electric fields and cell plasticity because ion channels, together with ion pumps and gap junction complexes, play a crucial role in cell communication and control. ${ }^{13-16}$ In this Letter, we present measurements and model calculations of the rectifying properties of a biological ion channel (the outer membrane protein $\mathrm{F}, \mathrm{OmpF}$, a porin expressed in Escherichia coli bacteria ${ }^{17}$ ). We consider the $\mathrm{pH}$-controlled net current induced by zero-average oscillating potentials and the electrical pumping of potassium ions against an external concentration gradient, a phenomenon previously reported in synthetic nanopores. ${ }^{4,5}$ We show that the complex interplay between the current rectification and the channel ionic selectivity is crucial for understanding the uphill transport of ions. The results are of relevance for the mechanisms regulating the transport through ion channels, ${ }^{17-19}$ for electric signal transduction in cell cycle and embryogenesis, ${ }^{14,16}$ and for building ionic circuits in the emerging field of nanofluidics. ${ }^{6,20-25}$

The set-up used in the experiments is shown schematically in Fig. 1. A single OmpF ion channel is reconstituted on a planar lipid bilayer and communicates the two halves of a conductivity cell filled with $\mathrm{KCl}$ solutions of concentrations $c_{c i s}$ (side of protein addition) and $c_{\text {trans }} . p H_{c i s}$ and $p H_{\text {trans }}$ denote the corresponding $p H$ values. A detailed description of the reconstitution procedure can be found elsewhere. ${ }^{17,26}$ The electric potential $V$ is positive when it is higher at the trans side of the membrane cell. An Axopatch 200B amplifier (Molecular Devices, Sunnyvale, CA) in the

\footnotetext{
${ }^{\text {a) }}$ Author to whom correspondence should be addressed. Electronic mail: alcaraza@uji.es
}

voltage-clamp mode is used for measuring both $V$ and the electric current $(I)$ through the channel. $I$ is positive when it flows from solution trans to solution cis in Fig. 1.

The OmpF porin contains both basic (positive) and acidic (negative) residues, ${ }^{27}$ and thus, the net charge of the channel depends on the ionization state of the protein residues. The ionic selectivity of the channel (preference for cations or anions) depends on many factors (electrolyte type and concentration, lipid charge, etc.) being the solution $p H$

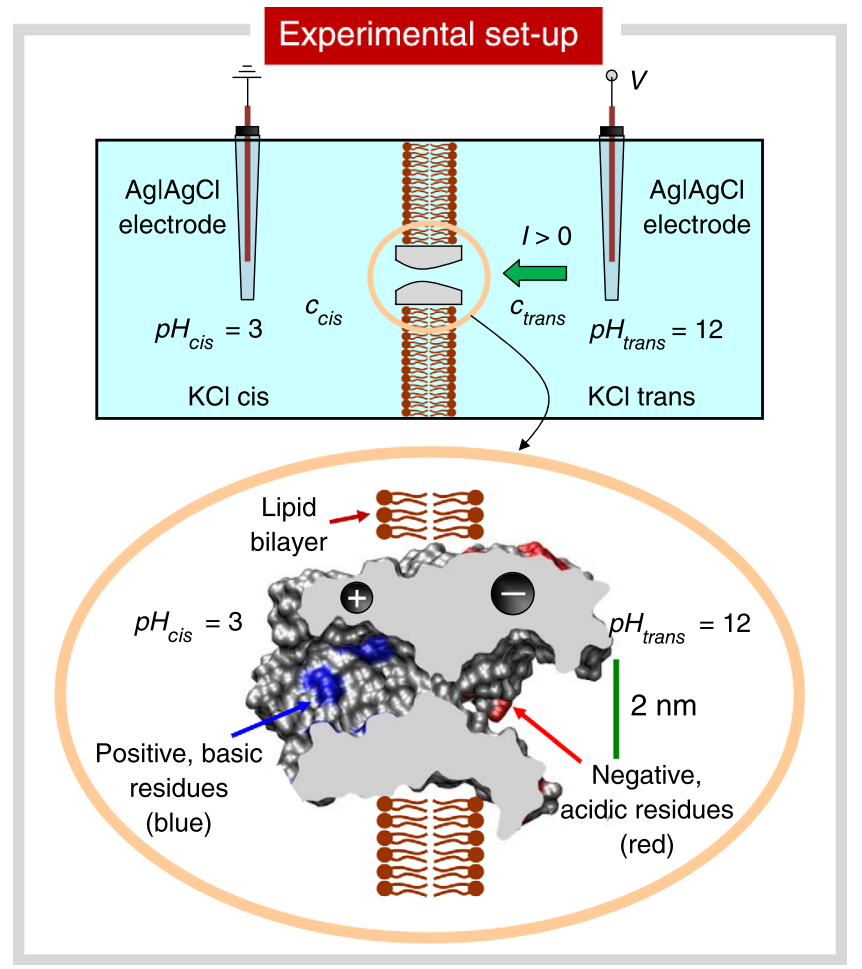

FIG. 1. Scheme of the experimental set-up. A single OmpF porin is reconstituted on a planar lipid bilayer and communicates two $\mathrm{KCl}$ solutions of concentrations $c_{c i s}$ and $c_{\text {trans }}$, with $p H_{c i s}=3$ and $p H_{\text {trans }}=12$. The $I$ - $V$ curves are measured with $\mathrm{Ag} \mid \mathrm{AgCl}$ electrodes. The asymmetric $\mathrm{pH}$ conditions lead to a non-homogeneous fixed charge distribution in the porin that shows rectifying characteristics in the current-voltage $(I-V)$ curves. 
the most significant one. ${ }^{26}$ At low $p H$, the channel is positively charged and selective to anions, whereas at high $\mathrm{pH}$, the channel is negatively charged and selective to cations. ${ }^{26,28}$ For $p H$ values close to neutrality, the channel exhibits a slight selectivity to cations. We have shown previously that not only the ion selectivity but also the $I-V$ characteristics of OmpF depend strongly on the $\mathrm{pH}$ of the external solutions. ${ }^{17,18}$ Under symmetrical conditions $\left(p H_{\text {cis }}=p H_{\text {trans }}\right.$ ), the experiments show linear, ohmic $I-V$ curves. Asymmetric configurations $\left(p H_{\text {cis }} \neq p H_{\text {trans }}\right)$ lead to nonlinear, rectifying $I-V$ curves. ${ }^{17}$ In particular, for $p H_{c i s}=3$ and $p H_{\text {trans }}=12$ (Fig. 1), the channel side facing the left solution is positively charged, while the opposite side has a negative fixed charge. ${ }^{17,18}$ This asymmetric structure constitutes a nanofluidic diode similar to solid state $\mathrm{p}$-n junctions and bipolar membranes. ${ }^{29}$

In order to promote the uphill transport of $\mathrm{K}^{+}$ions, we apply a zero-average time dependent potential to the $\mathrm{OmpF}$ channel in the configuration of Fig. 1. Fig. 2 describes the principles of the proposed mechanism. The concentration of the solutions in the experiment are $c_{c i s}=0.10 \mathrm{M}$ and $c_{\text {trans }}=0.05 \mathrm{M}$. Because of $c_{\text {cis }} \neq c_{\text {trans }}$, the ions flow from the concentrated solution towards the diluted one giving rise

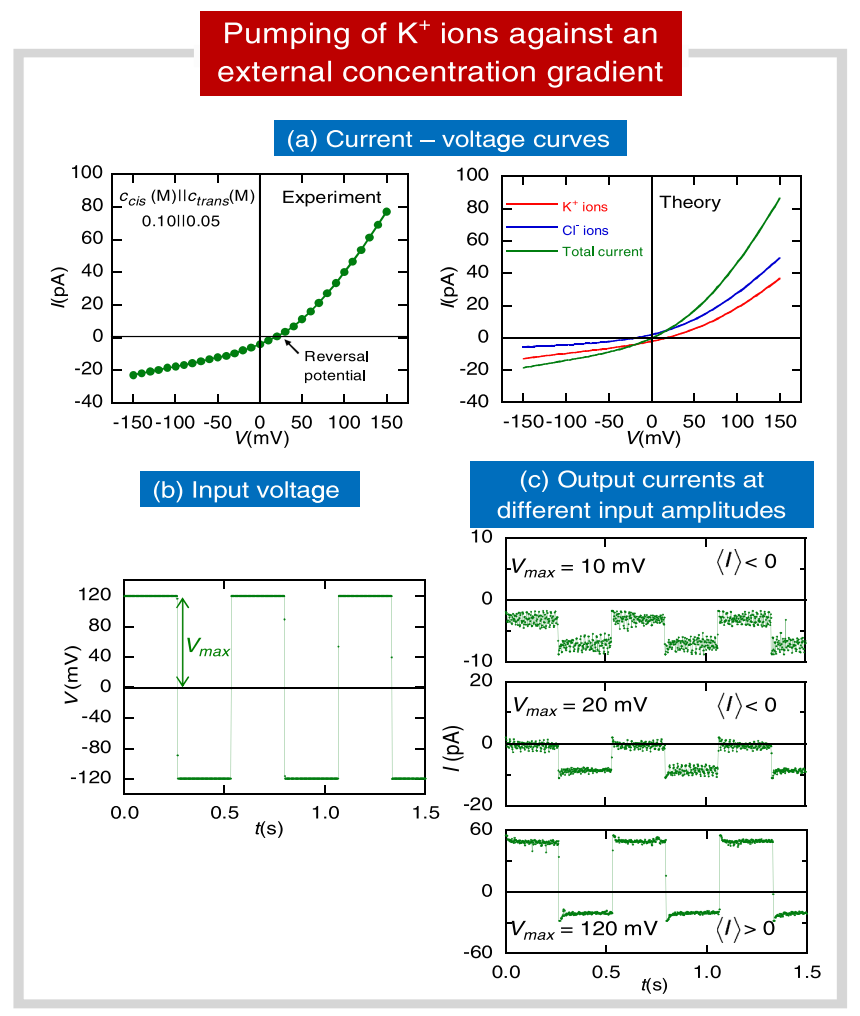

FIG. 2. Mechanism of pumping of $\mathrm{K}^{+}$ions against an external concentration gradient. (a) The DC $I-V$ characteristics of the $\mathrm{OmpF}$ channel, measured under the $\mathrm{pH}$ and $\mathrm{KCl}$ configurations indicated in the left panel, show rectification. The theoretical predictions of a PNP model based on the threedimensional structure of the channel (right panel) give the current carried by each ion. The input voltage signal used as driving force (b) has zero time average and amplitude $V_{\max }$. The average value $\langle I\rangle$ of the output current (c) increases with $V_{\max }$. At high enough $V_{\max }$ values (rectification ratio $r>1$ in the $I-V$ curve), $\langle I\rangle$ becomes positive. In the case of an ideally cationselective pore, $\langle I\rangle>0$ gives a net uphill transport of $\mathrm{K}^{+}$ions from the dilute solution to the concentrated one. Since the OmpF channel is weakly selective, the transport of $\mathrm{Cl}^{-}$ions across the channel is significant and the condition $\langle I\rangle>0$ is not sufficient for the uphill transport of $\mathrm{K}^{+}$ions. to an electrical net current without any applied voltage. The electric potential difference needed to obtain a zero current (the so called reversal potential, $V_{\text {rev }}$ ) is positive in this case, meaning that the channel exhibits a certain preference for cations. ${ }^{18}$ The DC I-V characteristics of the channel measured under these $\mathrm{pH}$ and $\mathrm{KCl}$ configurations (left panel of Fig. 2(a)) shows rectification, with a high conductance state for $V>0$ and a low conductance state for $V<0 .{ }^{17}$

The right panel of Fig. 2(a) shows the theoretical predictions of a Poisson-Nernst-Planck (PNP) model based on the three-dimensional structure of the OmpF porin (Protein Data Bank id. code: 2OMF) obtained from X-ray analysis. ${ }^{27}$ The fixed charge distribution is obtained from the 3D structure and free solution ionic diffusion coefficients are used. ${ }^{30}$ The model calculations show the total current (green) and the individual currents carried by each ion $\left(\mathrm{K}^{+}\right.$in red and $\mathrm{Cl}^{-}$in blue). Positive values of $I_{K}$ and negative values of $I_{C l}$ correspond, respectively, to uphill transport of the respective ion. As the input driving force, we use square-wave potentials $V(t)$ of zero time average (Fig. 2(b)) and amplitude $V_{\max }$. The output is the electric current $I(t)$ passing through the channel (Fig. $2(\mathrm{c}))$. Because the period of the applied voltage wave $(\sim 0.5 \mathrm{~s})$ is much longer than the characteristic time for axial diffusion in the channel $\tau=L^{2} / D_{i} \approx 10^{-7}-10^{-6} \mathrm{~s}(L \approx 5 \mathrm{~nm}$ is the length of the channel and $D_{i} \approx 10^{-9}-10^{-8} \mathrm{~m}^{2} / \mathrm{s}$ is the ionic diffusion coefficient), the output current is slave of the input signal. ${ }^{7}$ The maximum and minimum values of $I(t)$ at a given signal amplitude $V_{\max }$ correspond to those measured in the DC $I-V$ curve at $V=V_{\max }$ and $V=-V_{\max }$, respectively. We assume that, for the square input signal used here, the average current $\langle I\rangle$ can be written as follows: ${ }^{31}$

$$
\langle I\rangle=\frac{I\left(V_{\max }\right)+I\left(-V_{\max }\right)}{2}=\left|I\left(-V_{\max }\right)\right| \frac{(r-1)}{2},
$$

where $r \equiv\left|I\left(V_{\max }\right) / I\left(-V_{\max }\right)\right|$ is the current ratio of the channel at $V=V_{\max }$. Fig. 2(c) shows the dependence of the output current on the amplitude of the input signal. For $V_{\max }$ $\left\langle V_{\text {rev }}\right.$, both $I\left(V_{\max }\right)$ and $I\left(-V_{\max }\right)$ are negative, and then $\langle I\rangle$ is also negative. For signal amplitudes $V_{\max }$ slightly larger than $V_{r e v}$, the channel responds with $I\left(V_{\max }\right)>0$, but the amplitude of the driving force still cannot achieve $\langle I\rangle>0$ because $\left|I\left(V_{\max }\right)\right|<\left|I\left(-V_{\max }\right)\right|$. Eq. (1) reveals that the input amplitude $V_{0}$ needed to obtain $\langle I\rangle>0$ is that corresponding to $r>1$ in the $I-V$ curve where $\left|I\left(V_{\max }\right)\right|>\left|I\left(-V_{\max }\right)\right|$. Current rectification and non-linearity of the $I-V$ curves are necessary to obtain an uphill transport. However, we should find out whether this condition is sufficient or other requirements must be accomplished to pump ions against the external concentration gradient.

In the case of a perfect, ideally cation-selective pore (impermeable to anions), positive values of the average output current result in a net uphill transport of $\mathrm{K}^{+}{ }^{4}$ However, the $O m p F$ channel is only partially selective to cations, ${ }^{26,28}$ as it is the case of most ion channel and nanopores. The fact that both cations and anions contribute to the overall current in a proportion which could change considerably with the applied voltage has not been addressed previously. In the right panel of Fig. 2(a), we obtain $I<0$ for $V=0$ because the negative current $I_{K}$ is larger than the positive $I_{C l}$, being 

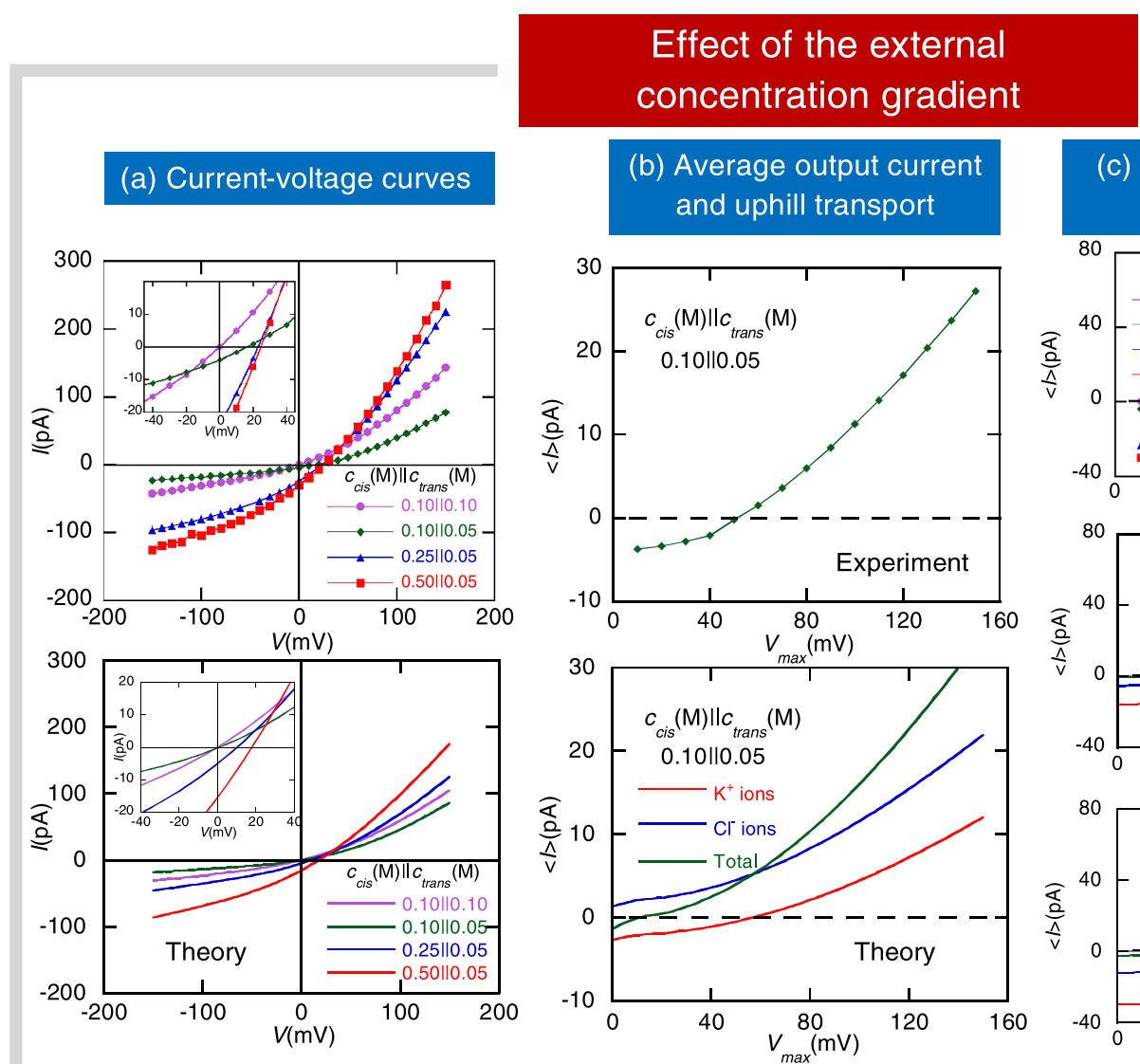

(b) Average output current and uphill transport

(c) Average output current and concentration gradient
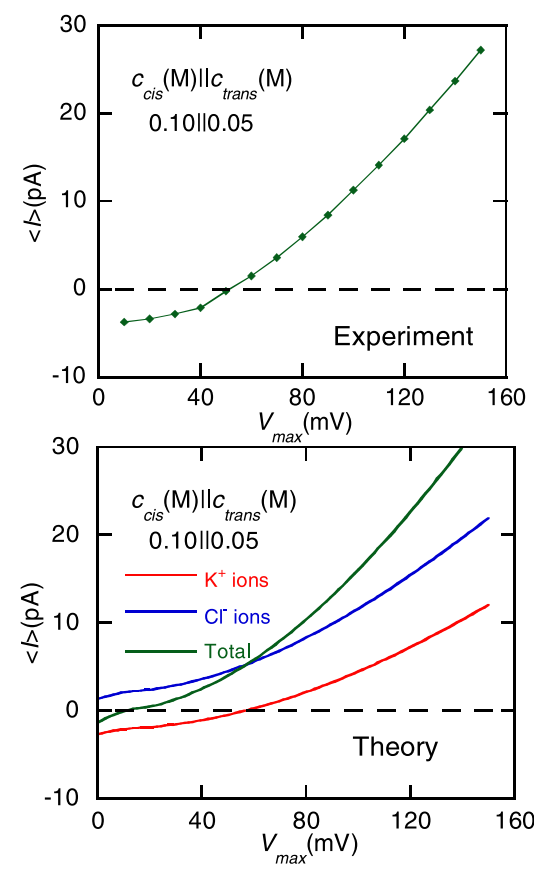
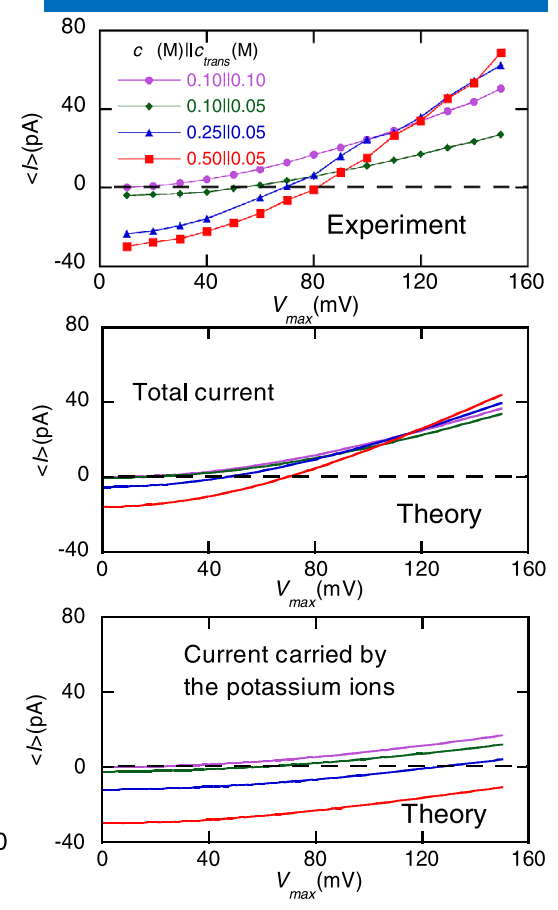

FIG. 3. Influence of the external concentration gradient on the pumping effect. (a) Experimental $I-V$ curves (upper panel) and theoretical predictions of the PNP model under the conditions indicated (lower panel). (b) Experimental curve of $\langle I\rangle$ vs $V_{\max }$ (upper panel) and theoretical predictions for $\langle I\rangle$ and the average of the individual currents carried by the $\mathrm{K}^{+}$and $\mathrm{Cl}^{-}$ions (lower panel) in the case $c_{c i s}=0.10 \mathrm{M}$ and $c_{\text {trans }}=0.05 \mathrm{M}$. The model shows that the contribution of the $\mathrm{Cl}^{-}$ions to the average total current is even higher than that of the $\mathrm{K}^{+}$ions and thus the condition $r>1$ is not appropriate to characterize the uphill transport. (c) Experimental (upper panel) and theoretical (middle panel) plots of $\langle I\rangle$ vs $V_{\max }$ for different concentration gradients. The contribution of the $\mathrm{K}^{+}$ions to $\langle I\rangle$ is shown in the lower panel. Because of the weak selectivity to $\mathrm{K}^{+}$ions exhibited by the channel, $\langle I\rangle>0$ is not a sufficient condition for the uphill transport of these ions to occur, especially for high concentration gradients.

downhill both fluxes. For $V \approx V_{\text {rev }}$ (zero current), the fluxes are still downhill but now $I_{K} \approx I_{C l}$. However, for $V \approx V_{\text {Nernst }}$, $I_{K}=0$ and the electric current is exclusively carried by $\mathrm{Cl}^{-}$ ions. When $V>V_{\text {Nernst }}$ the channel displays uphill transport of cations and downhill transport of anions. For negative voltages, we have a different scenario because the transport of cations is always downhill and the transport of anions is reversed to uphill conditions only when $V<-V_{\text {Nernst }}$. This means that $\langle I\rangle$ reflects a complicated balance between the uphill transport of $\mathrm{K}^{+}$for $V>0$ and the downhill $\mathrm{K}^{+}$transport for $V<0$, in which the rectification properties of the $I-V$ curve become essential and the contribution of $\mathrm{Cl}^{-}$is not trivial.

Fig. 3 illustrates the effect of the concentration gradient on the $I-V$ curves together with the theoretical predictions of the PNP model under the same conditions (Fig. 3(a)). The model describes adequately how the electrical current increases with the ratio $c_{c i s} / c_{\text {trans }}$ for the different voltage polarities. Fig. 3(b) shows the change of $\langle I\rangle$ with $V_{\max }$ under the same conditions as in Fig. $2\left(c_{c i s}=0.10 \mathrm{M}\right.$ and $\left.c_{\text {trans }}=0.05 \mathrm{M}\right)$. The upper panel shows the experimental curve and the lower panel the theoretical calculations for the total current as well as the individual currents carried by each ion. The model results emphasize that, in a weak selective channel like OmpF, the condition $\langle I\rangle>0$ is not sufficient to assure the pumping of potassium ions against the external concentration gradient. Due to the considerable transport of $\mathrm{Cl}^{-}$ions, the condition $r>1$ in Eq. (1) results inadequate to characterize the uphill transport. The asymmetric conduction is required but the channel discrimination between cations and anions is also important. This discrimination is strongly dependent on the screening of the channel charges exerted by the electrolyte, as it is shown in Fig. 3(c). The experimental (upper panel) and theoretical (middle panel) $\langle I\rangle$ vs $V_{\max }$ curves are shown for different concentration gradients. The larger the concentration ratio, the higher the value of $V_{\max }$ needed to achieve $\langle I\rangle>0$, what shows that concentrated solutions screen the channel charges, thus decreasing the channel selectivity and the ability to rectify the current. This is clearly seen in the lower panel of Fig. $3(\mathrm{c})$, which shows the average $\mathrm{K}^{+}$current as a function of $V_{\max }$. The voltage needed to produce the uphill transport of $\mathrm{K}^{+}$ions is significantly higher than the value deduced from the condition $\langle I\rangle>0$.

In conclusion, the experiments of Figs. 2 and 3 suggest that the $\mathrm{OmpF}$ porin under asymmetric $\mathrm{pH}$ conditions can be used to pump potassium ions against an external concentration gradient. These experimental findings are similar to 
those obtained previously in synthetic nanopores and emphasize that the interaction between mobile carriers and fixed charges plays a central role in transport processes in mesoscopic channels. However, we have shown here that the deviations from ideal selectivity make the condition $\langle I\rangle>0$ necessary but not sufficient to assure the pumping of ions. A detailed analysis of the rectification properties and the channel discrimination between ions has proved necessary to rationalize this complex transport phenomenon. $\mathrm{pH}$ and electrolyte concentration gradients are central to cell biophy$\operatorname{sics}^{14,16,17,19}$ and the results obtained suggest also some basic mechanisms for uphill transport and signal transduction through biological ion channels.

The authors acknowledge Marcel Aguilella-Arzo and Vicente Gomez for assistance in the preparation of the artwork. Financial support from the Generalitat Valenciana (Project PROMETEO/GV/0069), the Ministry of Economy and Competitiveness of Spain (Project Nos. FIS2010-19810 and MAT2012-32084), Fundació Caixa Castelló-Bancaixa (Project No. P1-1B2012-03), and FEDER is also acknowledged.

${ }^{1}$ R. D. Astumian and P. Hänggi, Phys. Today 55(11), 33 (2002).

${ }^{2}$ A. Ajdari and J. Prost, C. R. Acad. Sci. II 315, 1635 (1992).

${ }^{3}$ D. R. Chialvo and M. M. Millonas, Phys. Lett. A 209, 26 (1995).

${ }^{4}$ Z. Siwy and A. Fulinski, Phys. Rev. Lett. 89, 198103(2002).

${ }^{5}$ Z. Siwy and A. Fulinski, Am. J. Phys. 72, 567 (2004).

${ }^{6}$ P. Ramirez, M. Ali, W. Ensinger, and S. Mafe, Appl. Phys. Lett. 101, 133108 (2012).

${ }^{7}$ C. Verdia-Baguena, M. Queralt-Martin, V. M. Aguilella, and A. Alcaraz, J. Phys. Chem. C 116, 6537 (2012).

${ }^{8}$ J. Cervera, B. Schiedt, R. Neumann, S. Mafe, and P. Ramirez, J. Chem. Phys. 124, 104706 (2006).

${ }^{9}$ J. Cervera, A. Alcaraz, B. Schiedt, R. Neumann, and P. Ramiirez, J. Phys. Chem. C 111, 12265 (2007).
${ }^{10}$ G. A. Griess, E. Rogers, and P. Serwer, Electrophoresis 22, 981 (2001).

${ }^{11}$ J. C. T. Eijkel and A. van den Berg, Microfluid Nanofluid 1, 249 (2005).

${ }^{12}$ J. Cervera, P. Ramirez, S. Mafe, and P. Stroeve, Electrochim. Acta 56, 4504 (2011).

${ }^{13}$ R. D. Astumian, J. C. Weaver, and R. K. Adair, Proc. Natl. Acad. Sci. U.S.A. 92, 3740 (1995).

${ }^{14}$ D. J. Blackiston, K. A. McLaughlin, and M. Levin, Cell Cycle 8, 3527 (2009).

${ }^{15}$ J. A. Manzanares, J. Cervera, and S. Mafe, Appl. Phys. Lett. 99, 153703 (2011).

${ }^{16}$ M. Levin and C. G. Stevenson, Annu. Rev. Biomed. Eng. 14, 295 (2012).

${ }^{17}$ A. Alcaraz, P. Ramirez, E. Garcia-Gimenez, M. L. Lopez, A. Andrio, and V. M. Aguilella, J. Phys. Chem. B 110, 21205 (2006).

${ }^{18}$ E. Garcia-Gimenez, A. Alcaraz, V. M. Aguilella, and P. Ramirez, J. Membr. Sci. 331, 137 (2009).

${ }^{19} \mathrm{~B}$. Hille, Ion Channels of Excitable Membranes (Sinauer Associates, Sunderland, 2001).

${ }^{20} \mathrm{H}$. Miedema, M. Vrouenraets, J. Wierenga, W. Meijberg, G. Robillard, and B. Eisenberg, Nano Lett. 7, 2886 (2007).

${ }^{21}$ C. Dekker, Nature Nanotech. 2, 209 (2007).

${ }^{22}$ G. Maglia, A. J. Heron, W. L. Hwang, M. A. Holden, E.Mikhailova, Q. Li, S.Cheley, and H. Bayley, Nat. Nanotechnol. 4, 437-440 (2009).

${ }^{23}$ E. O. Gabrielsson, K. Tybrandt, and M. Berggren, Lab Chip 12, 2507 (2012).

${ }^{24}$ M. X. Macrae, S. Blake, X. Jiang, R.Capone, D. J. Estes, M. Mayer, and J. Yang, ACS Nano 3, 3567 (2009).

${ }^{25}$ N. A. Wilson, R. Abu-Shumays, B. Gyarfas, H. Wang, K. R. Lieberman, M. Akeson, and W. B. Dunbar, ACS Nano 3, 995-1003 (2009).

${ }^{26}$ A. Alcaraz, E. M. Nestorovich, M. Aguilella-Arzo, V. M. Aguilella, and S. M. Bezrukov, Biophys. J. 87, 943 (2004).

${ }^{27}$ S. W. Cowan, T. Schirmer, G. Rummel, M. Steiert, R. Ghosh, R. A. Pauptit, J. N. Jansonius, and J. P. Rosenbusch, Nature 358, 727 (1992).

${ }^{28}$ E. M. Nestorovich, T. K. Rostovtseva, and S. M. Bezrukov, Biophys. J. 85, 3718 (2003)

${ }^{29}$ A. Alcaraz, P. Ramirez, S. Mafe, H. Holdik, and B. Bauer, Polymer 41, 6627 (2000).

${ }^{30}$ M. Aguilella-Arzo, J. J. García-Celma, J. Cervera, A. Alcaraz, and V. M. Aguilella, Bioelectrochem. 70, 320 (2007).

${ }^{31}$ P. Ramirez, V. Gomez, M. Ali, W. Ensinger, and S. Mafe, Electrochem. Commun. 31, 137 (2013). 